\title{
Evidence for biquadratic exchange in the quasi-two-dimensional antiferromagnet FePS 3
}

Cite as: J. Appl. Phys. 127, 223903 (2020); doi: 10.1063/5.00091 14

Submitted: 27 March 2020 . Accepted: 24 May 2020 .

Published Online: 9 June 2020

A. R. Wildes, ${ }^{1, a)}$ (D) M. E. Zhitomirsky, ${ }^{2}$ (D) T. Ziman, ${ }^{1,3}$ (D) D. Lançon, ${ }^{1,4, b) ~(D) ~ a n d ~ H . ~ C . ~ W a l k e r ~}{ }^{5}$ (D)

\section{AFFILIATIONS}

${ }^{1}$ Institut Laue-Langevin, 71 avenue des Martyrs CS 20156, 38042 Grenoble Cedex 9, France

${ }^{2}$ Univ. Grenoble Alpes, CEA, IRIG, PHELIQS, 17 avenue des Martyrs, 38000 Grenoble, France

${ }^{3}$ Univ. Grenoble Alpes, CNRS, LPMMC, 38000 Grenoble, France

${ }^{4}$ Ecole Polytechnique Fédérale de Lausanne, SB ICMP LQM, CH-1015 Lausanne, Switzerland

${ }^{5}$ ISIS Facility, Rutherford Appleton Laboratory, Harwell Oxford, Didcot OX11 OQX, United Kingdom

Note: This paper is part of the Special Topic on 2D Quantum Materials: Magnetism and Superconductivity

a) Author to whom correspondence should be addressed: wildes@ill.fr

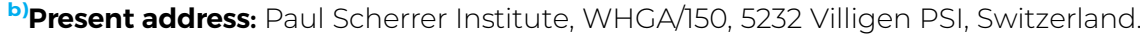

\begin{abstract}
$\mathrm{FePS}_{3}$ is a van der Waals compound with a honeycomb lattice that is a good example of a two-dimensional antiferromagnet with Ising-like anisotropy. Neutron spectroscopy data from $\mathrm{FePS}_{3}$ were previously analyzed using a straightforward Heisenberg Hamiltonian with a single-ion anisotropy. The analysis captured most of the elements of the data; however, some significant discrepancies remained. The discrepancies were most obvious at the Brillouin zone boundaries. The data are subsequently reanalyzed, allowing for unequal exchange between nominally equivalent nearest-neighbors, which resolves the discrepancies. The source of the unequal exchange is attributed to a biquadratic exchange term in the Hamiltonian, which most probably arises from a strong magnetolattice coupling. The new parameters show that there are features consistent with Dirac magnon nodal lines along certain Brillouin zone boundaries.
\end{abstract}

Published under license by AIP Publishing. https://doi.org/10.1063/5.0009114

\section{INTRODUCTION}

$\mathrm{FePS}_{3}$ belongs to a family of layered van der Waals compounds. It is monoclinic with space group $C \frac{2}{m}$ and lattice parameters $a=5.947 \AA, b=10.3 \AA, c=6.7222 \AA$, and $\beta=107.16^{\circ}{ }^{1}$. The $\mathrm{Fe}^{2+}$ ions are located at fractional coordinates $(0,0.3326,0)$, forming a slightly distorted honeycomb lattice in the $a b$ planes. The compound is antiferromagnetic, ordering below its Néel temperature of $\sim 120 \mathrm{~K}^{2,3}$ with a propagation vector of $\mathbf{k}_{M}=\left(01 \frac{1}{2}\right)$., The high-spin $(S=2) \mathrm{Fe}^{2+}$ are aligned parallel to the $\mathrm{c}^{*}$ direction, i.e., normal to the $a b$ planes, forming ferromagnetic zig-zag chains that are parallel to the $a$ axis. The chains are antiferromagnetically coupled along the $b$ and $c$ axes. Figure 1(a) shows a schematic of the magnetic structure in the $a b$ planes. The paramagnetic susceptibility is highly anisotropic, ${ }^{3}$ and the compound appears to be a good example of an Ising-like antiferromagnet.

Other transition metals with $2+$ valence may be substituted for iron, and the transition metal-PS $\mathrm{P}_{3}$ family of compounds has attracted considerable attention over the years. ${ }^{6-9}$ The weak van der Waals forces binding the $a b$ planes allow for intercalation with a wide variety of materials including lithium, ${ }^{6,7}$ and the compounds have been heavily investigated as prospects for battery materials. The compounds are insulators, but they can be driven to become metallic on the application of pressure ${ }^{10}$ providing avenues for fundamental studies of correlated electron physics. One related compound, $\mathrm{FePSe}_{3}$, can even be driven into a superconducting state at sufficiently high pressure. ${ }^{11}$ The weak binding also extends to the magnetic coupling between the $a b$ planes, and the family are model systems for the study of low-dimensional magnetism. $\mathrm{FePS}_{3}$ is particularly interesting in this regard due to its Ising-like nature. As shown by Onsager, ${ }^{12}$ long-ranged magnetic order is allowed in the two-dimensional Ising model, helping to explain the relatively high Néel temperature in $\mathrm{FePS}_{3}$. The discovery of graphene has led to a search for other compounds that may be delaminated to monolayer thickness. The transition metal- $\mathrm{PS}_{3}$ compounds are 

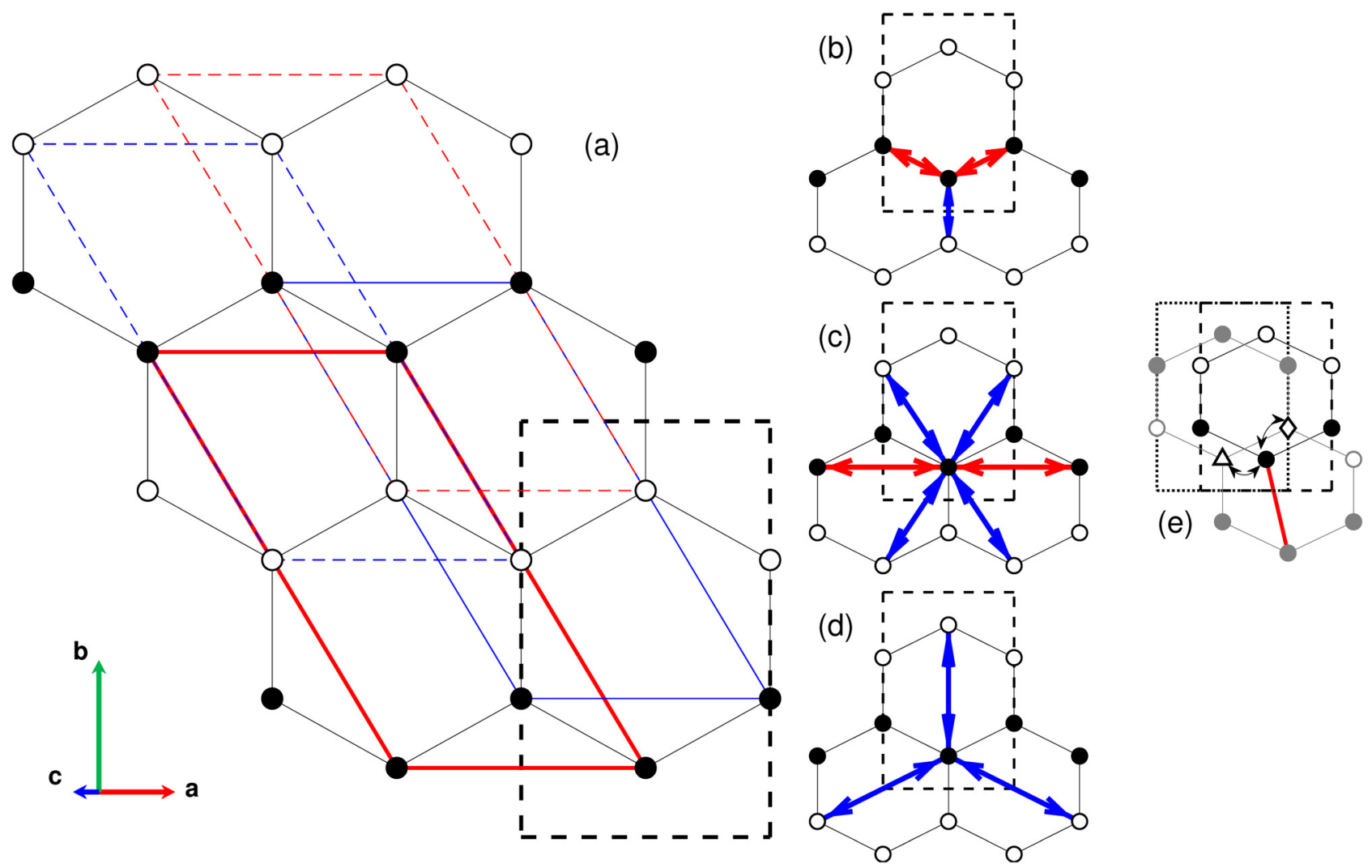

FIG. 1. The magnetic structure and exchange parameters of $\mathrm{FePS}_{3}$, as viewed along the $\mathbf{c}^{*}$ axis. Open and closed symbols show moments into and out of the page, respectively. The monoclinic unit cell in the ab planes is shown as black dashed lines. (a) The magnetic structure in the $a b$ planes. The magnetic structure may be decomposed into four interlocking Bravais lattices, shown as red and blue solid and dashed lines. (b) The nearest-neighbor exchanges, $J_{1, \alpha}$ and $J_{1, \phi}$, indicated by blue and red arrows, respectively. (c) The second nearest-neighbor exchanges, $J_{2, \alpha}$ and $J_{2, \phi}$, indicated by blue and red arrows, respectively. (d) The third nearest-neighbor exchanges, $J_{3}$. (e) The interlayer exchanges. The moments shown as black circles are in an ab plane one unit cell below the symbols representing all other moments. The exchange $J^{\prime}$ is indicated by the arrow between the open triangle and the closed circle, and $J^{\prime \prime}$ is indicated by the arrow between the open diamond and the closed circle. The $\mathbf{c}_{\text {mag }}$ axis for the corresponding interlocking Bravais lattice shown in (a) is shown as a red solid line.

particularly interesting as they are magnetic. ${ }^{8,9}$ They can be delaminated, and $\mathrm{FePS}_{3}$ has been shown to maintain its Ising-like magnetic properties and antiferromagnetic ordering even for an isolated $a b$ plane, ${ }^{13}$ in agreement with the Onsager solution.

Neutron scattering has been used to measure the spin-wave dispersion, and the data were fitted using linear spin-wave theory by Lançon et $a l^{5}$ The spin-wave theory was based on a simple Heisenberg Hamiltonian with a single-ion anisotropy, and the honeycomb lattice was assumed to be perfect. The data showed that $\mathrm{FePS}_{3}$ was a good example of a two-dimensional antiferromagnet with very weak spin-wave dispersion between the $a b$ planes, and a large energy gap of $\sim 15 \mathrm{meV}$ explaining its Ising-like behavior. Satisfactory fits to the data required the inclusion of up to the third nearest-neighbor in the $a b$ planes. The fitting showed that the interplanar exchange was $\sim 1 / 200$ times the magnitude of the nearest-neighbor intraplanar exchange and a large anisotropy dominating the magnitude of the gap. However, there were some discrepancies between the calculations for the magnon dynamics and the measured neutron data. The discrepancies were most apparent along the Brillouin zone boundaries where the data appeared to be far more symmetric about the central energy in the dynamic band width than could be reproduced by the model.

The data from Lançon et al. have consequently been reanalyzed using a more general expression for the dynamic structure that allows for exchanges on nominally equivalent nearestneighbors to be different. The agreement with the data is significantly improved when fitting with this expression, although the results show large differences between the exchanges for nominally equivalent neighbors. The differences appear to be too large to be explained by the slight departure from having a perfect honeycomb structure. The differences are discussed in the context of adding a biquadratic exchange term, $\left(\mathbf{S}_{i} \cdot \mathbf{S}_{j}\right)^{2}$, to the spin Hamiltonian. 


\section{HAMILTONIANS AND THE DYNAMIC STRUCTURE FACTOR}

The Hamiltonian initially used to model the spin waves for $\mathrm{FePS}_{3}$ was given by the following equation:

$$
\hat{H}=-\sum_{<i j>} J_{i j} \mathbf{S}_{i} \cdot \mathbf{S}_{j}-\Delta \sum_{i}\left(S_{i}^{z}\right)^{2} .
$$

The dynamic structure factor was calculated using linear spin-wave theory. The magnetic structure was decomposed into four interlocking Bravais sublattices, as shown in Fig. 1(a). The axes for the magnetic Bravais lattices differ from those for the monoclinic unit cell, and the Miller indices for the two lattices are related via the transformation,

$$
\left[\begin{array}{l}
h \\
k \\
l
\end{array}\right]=\left[\begin{array}{lll}
1 & 0 & 0 \\
1 & 1 & 0 \\
0 & \frac{1}{2} & 1
\end{array}\right]\left[\begin{array}{l}
h_{\text {mag }} \\
k_{\text {mag }} \\
l_{\text {mag }}
\end{array}\right],
$$

where the subscript mag refers magnetic Miller indices and the absence of a subscript refers to the monoclinic Miller indices. The Holstein-Primakoff transformation was subsequently applied and, after Fourier transforming, the dynamic part of the Hamiltonian, $\mathbf{H}_{\mathbf{M}}$, could be expressed in the matrix form, ${ }^{5,14}$

$$
\mathbf{H}_{\mathbf{M}}=2 S\left[\begin{array}{cccc}
A & B^{*} & C & D^{*} \\
B & A & D & C \\
C & D^{*} & A & B^{*} \\
D & C & B & A
\end{array}\right] .
$$

The expressions for the matrix elements are given by the translation vectors between the different interlocking Bravais lattices. The honeycomb lattices were assumed to be perfect, which is exactly true for the $C \frac{2}{m}$ unit cell in the limits that $b=\sqrt{3} a$ and the fractional position for the magnetic ions being at $\left(0, \frac{1}{3}, 0\right)$. These conditions are very close to being met for $\mathrm{FePS}_{3}$. ${ }^{1}$ The matrix elements in Eq. (3) then become expressions with rational fractions,

$$
\begin{aligned}
& A=2 J_{2, \phi} \cos \left(2 \pi h_{\text {mag }}\right)-\Delta-2 J_{1, \phi}+J_{1, \alpha}-2 J_{2, \phi}+4 J_{2, \alpha}+3 J_{3}+2 J^{\prime}+2 J^{\prime \prime}, \\
& B=J_{1, \phi} \exp \left(\frac{2 \pi i}{3}\left[2 h_{\text {mag }}+\frac{k_{\text {mag }}}{2}\right]\right) \times\left(1+\exp \left(-2 \pi i h_{\text {mag }}\right)\right), \\
& C= 2 J_{2, \alpha}\left(\cos \left(\pi k_{\text {mag }}\right)+\cos \left(2 \pi\left[h_{\text {mag }}+\frac{k_{\text {mag }}}{2}\right]\right)\right)+J^{\prime} \cos \left(\pi\left[k_{\text {mag }}+2 l_{\text {mag }}\right]\right), \\
& D= \exp \left(\frac{2 \pi i}{3}\left[2 h_{\text {mag }}+\frac{k_{\text {mag }}}{2}\right]\right) \times\left\{\begin{array}{c}
J_{1, \alpha} \exp \left(-2 \pi i\left[h_{\text {mag }}+\frac{k_{\text {mag }}}{2}\right]\right) \\
+J_{3}\left(\begin{array}{c}
2 \cos \left(\pi k_{\text {mag }}\right) \\
+\exp \left(-2 \pi i\left[2 h_{\text {mag }}+\frac{k_{\text {mag }}}{2}\right]\right)
\end{array}\right)
\end{array}\right\} \\
&+2 J^{\prime \prime} \exp \left(\frac{\pi i}{3}\left[h_{\text {mag }}+k_{\text {mag }}\right]\right) \times \cos \left(\pi\left[h_{\text {mag }}+k_{\text {mag }}+2 l_{\text {mag }}\right]\right) .
\end{aligned}
$$

Figure 1(b) shows the exchange interactions between the nearest-neighbors of which there are three for a perfect honeycomb. None of these neighbors are on the same Bravais lattice as the initial magnetic ion with two of the neighbors on the ferromagnetically coupled Bravais lattice and the third on one of the two antiferromagnetically coupled Bravais lattices. The exchanges between these neighbors are defined as $J_{1, \phi}$ and $J_{1, \alpha}$ for the ferromagnetically and antiferromagnetically coupled moments shown as red and blue arrows in Fig. 1(b), respectively. Inspection of Fig. 1(c) shows that a similar situation exists for the second nearest-neighbors of which there are six on a perfect honeycomb. Two of the neighbors are on the same Bravais lattice as the initial ion. The interactions are shown as red arrows in the figure and they are defined as $J_{2, \phi}$. The other four neighbors are on the other antiferromagnetically coupled Bravais lattice, the exchanges are shown as the blue arrows and they are defined as $J_{2, \alpha}$. Figure 1 (d) shows the third nearestneighbor exchange, $J_{3}$, which are all between ions on the same antiferromagnetically coupled Bravais lattice.
Figure 1(e) shows the interplanar exchanges along with the $\mathbf{c}_{\text {mag }}$ axis for one of the magnetic Bravais lattices. There are two nearest-neighbors on an adjoining layer, one on each of the antiferromagnetically coupled Bravais lattices, and the two exchanges may be labeled as $J^{\prime}$ and $J^{\prime \prime}$. The interlayer distances to each of these nearest neighbors are identical in the limits of a perfect honeycomb lattice in the $a b$ planes and $a=-3 c \cos \beta$ for the $C \frac{2}{m}$ unit cell.

The expression for $\mathbf{H}_{\mathbf{M}}$ given in Eq. (3) must be multiplied by a matrix to respect the commutation relations for the raising and lowering operators used in the Holstein-Primakov transformation. The eigenvalues for the resulting matrix give the dispersion relations for the magnon energies, $\omega_{\mathbf{q}}$, through the expression

$$
\begin{aligned}
\omega_{\mathbf{q}}^{2}= & A^{2}+|B|^{2}-C^{2}-|D|^{2} \\
& \pm\left(4\left|A B^{*}-C D^{*}\right|^{2}-\left|B D^{*}-D B^{*}\right|^{2}\right)^{\frac{1}{2}},
\end{aligned}
$$



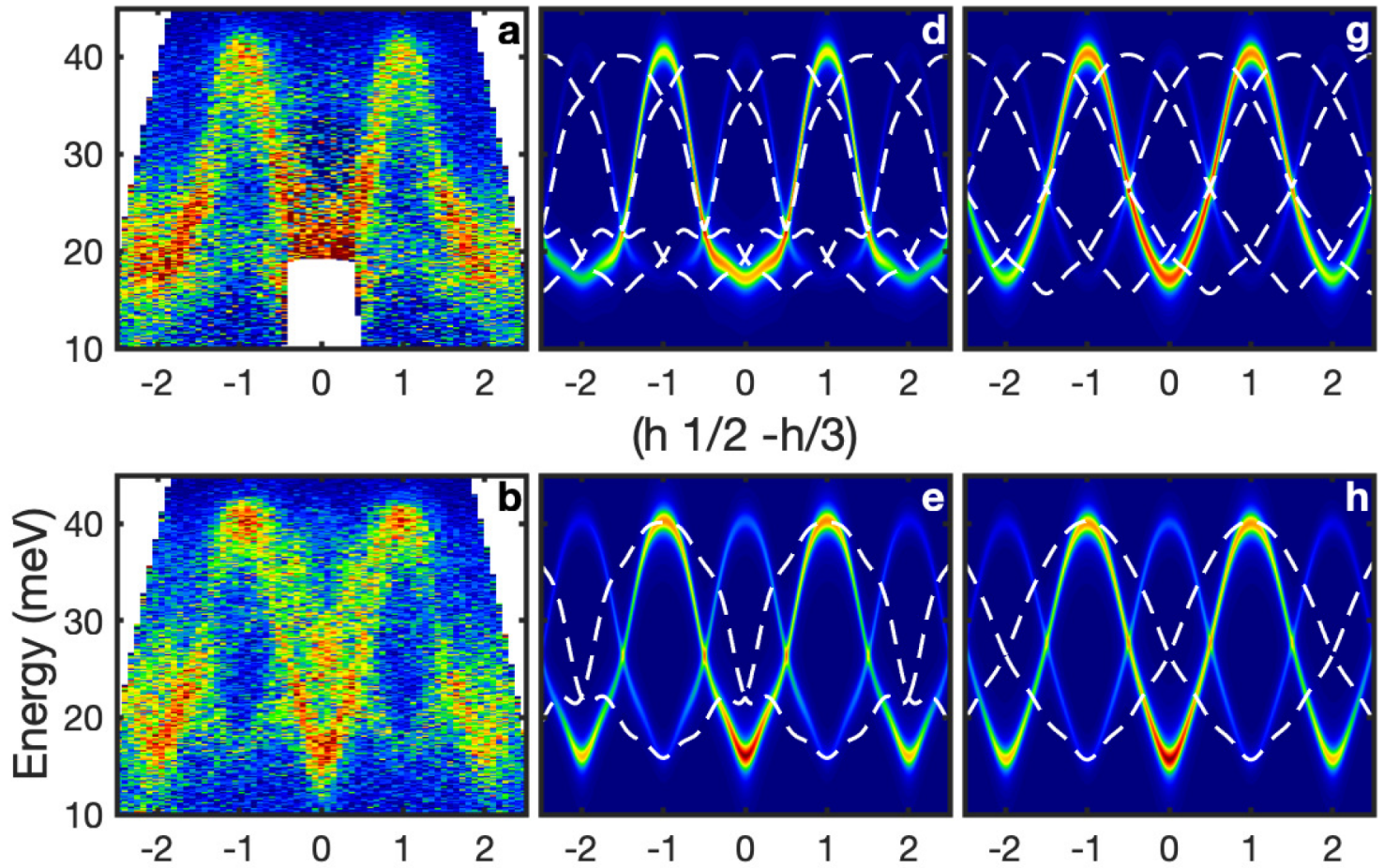

(h $1-\mathrm{h} / 3)$
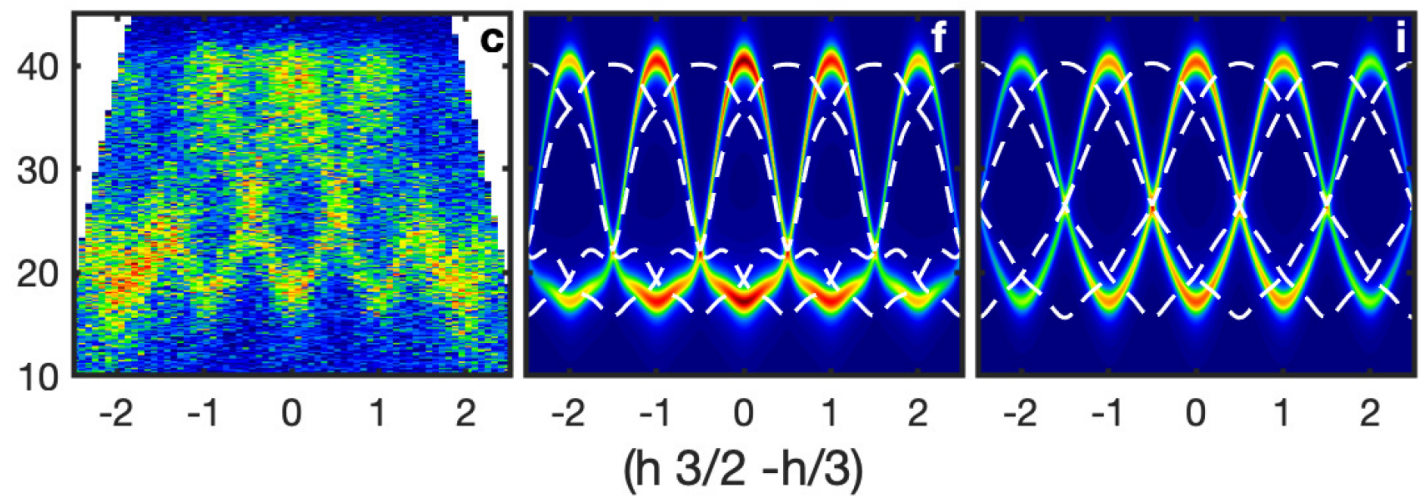

FIG. 2. Neutron scattering data and fit results for $S(\mathbf{Q}, \omega)$ along trajectories parallel to $\left(h 0 \frac{\bar{h}}{3}\right)$ for FePS$S_{3}$. (a)-(c) show the neutron scattering data measured at $5 \mathrm{~K}$ from the experiments performed by Lançon et al. ${ }^{5}$ (d)-(f) show the calculated $S(\mathbf{Q}, \omega)$ using the fitted parameters on constraining $J_{1, \phi}=J_{1, \alpha}$ and $J_{2, \phi}=J_{2, \alpha}$, following the analysis by Lançon et $\mathrm{al}^{5}{ }^{5} \mathrm{~g}$ )-(i) show the calculations from fits where the corresponding nearest-neighbor exchanges were not constrained to be equal. Trajectories centered at $\left(0 \frac{1}{2} 0\right)$ are shown in (a), (d), and $(\mathrm{g})$, trajectories centered at $(010)$ are show in $(\mathrm{b}),(\mathrm{e})$, and $(\mathrm{h})$ and trajectories centered at $\left(0 \frac{3}{2} 0\right)$ are shown in $(\mathrm{c}),(\mathrm{f})$, and $(\mathrm{i})$. The dashed lines in (d)-(i) show the calculated positions for the contributions from crystal twins. (a)-(f) are adapted with permission from Phys. Rev. B, 94, 214407 (2016). Copyright 2016 American Physical Society.

and the dynamic structure factor, $S_{\text {mag }}(\mathbf{Q}, \omega)$, is given by the normalized eigenvectors, ${ }^{14}$ which have been explicitly described for Eq. (3) by Wheeler et al. ${ }^{15}$

For a perfect two-dimensional honeycomb lattice, the nearestneighbor distances in Fig. 1(b) are equivalent and there is no reason for $J_{1, \phi}$ and $J_{1, \alpha}$ to be inequivalent. Equation (4) may be simplified by equating $J_{1, \phi}=J_{1, \alpha}=J_{1}$ and similarly $J_{2, \phi}=J_{2, \alpha}=J_{2}$. The zig-zag magnetic structure shown in Fig. 1 is stabilized due to the relative strengths of the three intraplanar exchange interactions, $J_{1}, J_{2}$, and $J_{3} .{ }^{16-18}$

The introduction of a biquadratic term into Eq. (1) causes the exchanges between different Bravais lattices to become inequivalent, 
i.e., $J_{1, \phi} \neq J_{1, \alpha}$ and $J_{2, \phi} \neq J_{2, \alpha}$. The Hamiltonian with biquadratic exchange takes the form, ${ }^{19,20}$

$$
\hat{H}=-\sum_{\langle i j\rangle}\left[J_{i j} \mathbf{S}_{i} \cdot \mathbf{S}_{j}-\tilde{K}_{i j}\left(\mathbf{S}_{i} \cdot \mathbf{S}_{j}\right)^{2}\right]-\Delta \sum_{i}\left(S_{i}^{z}\right)^{2} .
$$

In the limit of a collinear antiferromagnetic structure, the biquadratic coupling between neighbors, $\tilde{K}_{i j}$, may be incorporated into an effective exchange,

$$
\begin{gathered}
J_{i j, \phi} \rightarrow J_{i j}-2 \tilde{K}_{i j} S^{2}, \\
J_{i j, \alpha} \rightarrow J_{i j}+2 \tilde{K}_{i j} S^{2},
\end{gathered}
$$

where $J_{i j, \phi}$ describes the exchange between ferromagnetically coupled neighbors and $J_{i j, \alpha}$ is the exchange for antiferromagnetic neighbors. The substitution in Eq. (7) is the result of the second- order expansion of the biquadratic term around the antiferromagnetic structure in terms of the bosonic magnon operators. Consequently, it is fully applicable to harmonic spin-wave theory and can be used to determine correctly the magnon dispersion and the corresponding dynamic structure factor. Equation (3) preserves its matrix form with precisely the same matrix elements as given by Eq. (4). However, the substitution is not appropriate for classical energy calculations which must be performed using the full spin Hamiltonian in Eq. (6).

\section{FITTING THE NEUTRON SPECTROSCOPY DATA}

Lançon et $a l^{5}$ used the MERLIN time-of-flight neutron spectrometer at the ISIS Facility, UK to measure the magnetic scattering from $\mathrm{FePS}_{3}$ at $5 \mathrm{~K}$. The instrument captures a large volume of reciprocal space, and the data may subsequently be presented as

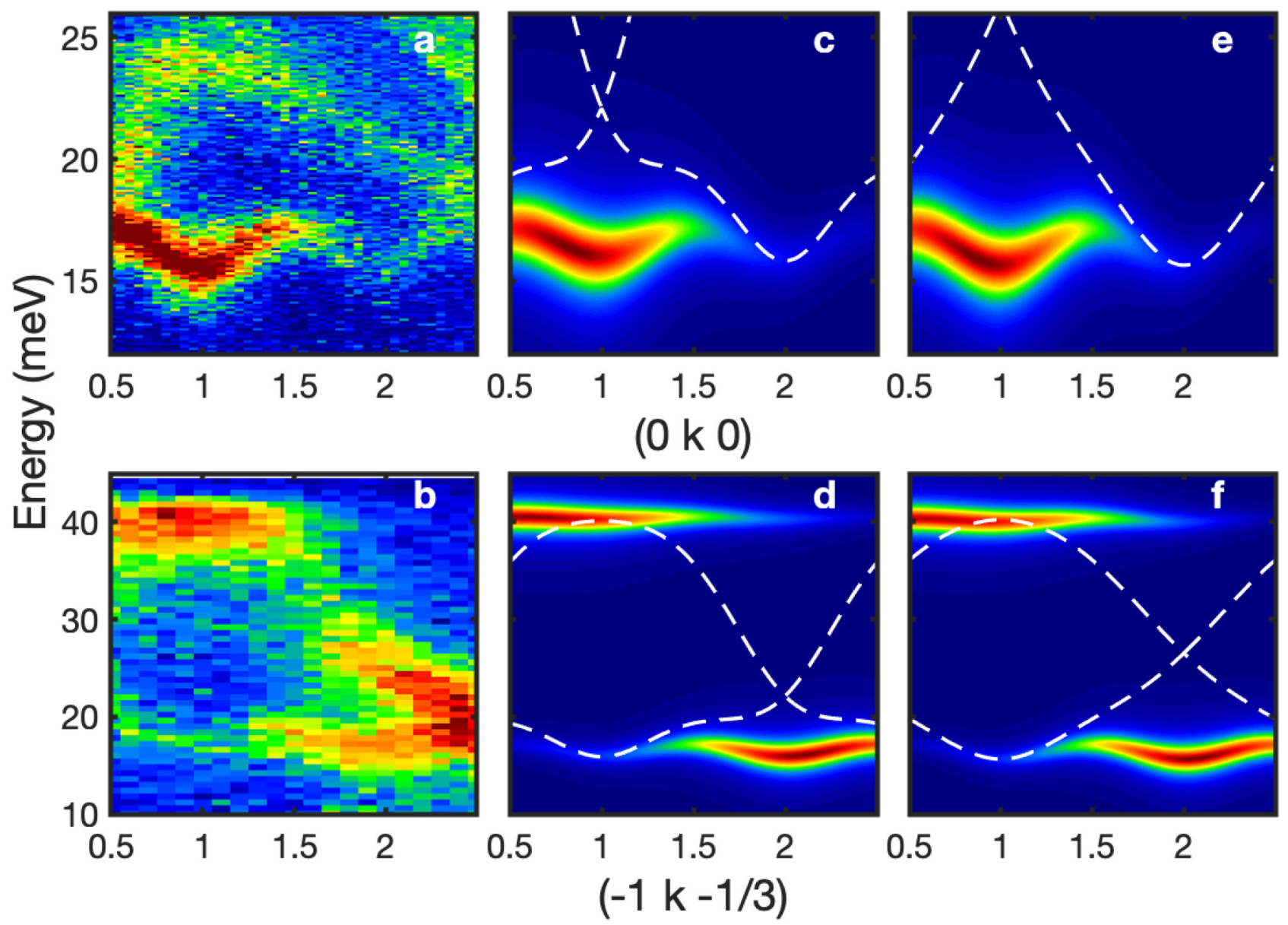

FIG. 3. Neutron scattering data and fit results for $S(Q, \omega)$ along trajectories parallel to $(0 k 0)$ for FePS 3 . (a) and (b) show the neutron scattering data measured at $5 \mathrm{~K}$ from the experiments performed by Lançon et al. ${ }^{5}$ (c) and (d) show the calculated $S(Q, \omega)$ using the fitted parameters on constraining $J_{1, \phi}=J_{1, \alpha}$ and $J_{2, \phi}=J_{2, \alpha}$, following the analysis by Lançon et al. ${ }^{5}$ (e) and (f) show the calculations from fits where the corresponding nearest-neighbor exchanges were not constrained to be equal. Trajectories along (0k0) are shown in (a), (c), and (e) and trajectories along $\left(\overline{1} k \frac{1}{3}\right)$ are show in (b), (d), and (f). The dashed lines in (c)-(f) show the calculated positions for the contributions from crystal twins. (a) and (c) are adapted with permission from Phys. Rev. B, 94, 214407 (2016). Copyright 2016 American Physical Society. 
slices. The data are shown in Figs. 2(a)-2(c), 3(a), and 3(b), corresponding to slices along different directions in the $a b$ planes. The inset of Fig. 4(d) shows a schematic for the Brillouin zone for a two-dimensional zig-zag structure on a honeycomb lattice. The $\mathbf{a}^{*}$ and $\mathbf{b}^{*}$ vectors are marked on the Brillouin zone for the $C \frac{2}{m}$ reciprocal lattice.

Figure 2 shows slices parallel to the monoclinic $\left(h 0 \frac{\bar{h}}{3}\right)$ direction. The slices are centered at different values of $k$, with Fig. 2(b) following the $\Gamma-\mathrm{Z}$ axis through Brillouin zone centers and Figs. 2(a) and 2(c) being slices along $\mathrm{Y}-\mathrm{C}$ zone boundaries. Figure 3 shows slices parallel to the $\mathbf{b}^{\star}$ axis, with Fig. 3(a) showing the data along the $\Gamma-\mathrm{Y}$ axis through Brillouin zone centers and Fig. 3(b) showing the slices along $\mathrm{Z}-\mathrm{C}$ Brilliouin zone boundaries. The data clearly show two intersecting spin-wave branches, as anticipated from Eq. (5). The branches are highly dispersive along the $\left(h 0 \frac{\bar{h}}{3}\right)$ direction and only weakly dispersive along directions parallel to the $\mathbf{b}^{*}$ axis. The data are complicated by the presence of twin domains prevalent in this family of compounds. ${ }^{21}$ The contributions from all the domains are superimposed in the data; however, they can be differentiated as they are quite distinct and are related by well-defined rotation matrices. ${ }^{5}$ The data also showed a small amount of spurious scattering, seen primarily in Fig. 3(a) as an arc of intensity descending from $\sim 25 \mathrm{meV}$ at $k=0.5$ to $\sim 18 \mathrm{meV}$ at $k=2.5$ and as a small amount of extra intensity in the top right-hand corner of the figure.

Spin-wave energies were extracted from Figs. 2(b), 2(c), and 3 (a) by Lançon et al. One-dimensional cuts were taken through the data, and the energies were determined by fitting damped harmonic oscillator functions, taking care to avoid or account for the spurious scattering and the contributions from other magnetic domains. The extracted spin-wave energies are shown in Figs. 4(a)-(c). The energies were then simultaneously fitted with Eq. (5) assuming that $J_{1}=J_{1, \phi}=J_{1, \alpha}, J_{2}=J_{2, \phi}=J_{2, \alpha}$, and $J^{\prime}=J^{\prime \prime}$, and the fits are shown as black lines in Figs. 4(a)-4(c). The exchange parameters for the three nearest intralayer neighbors
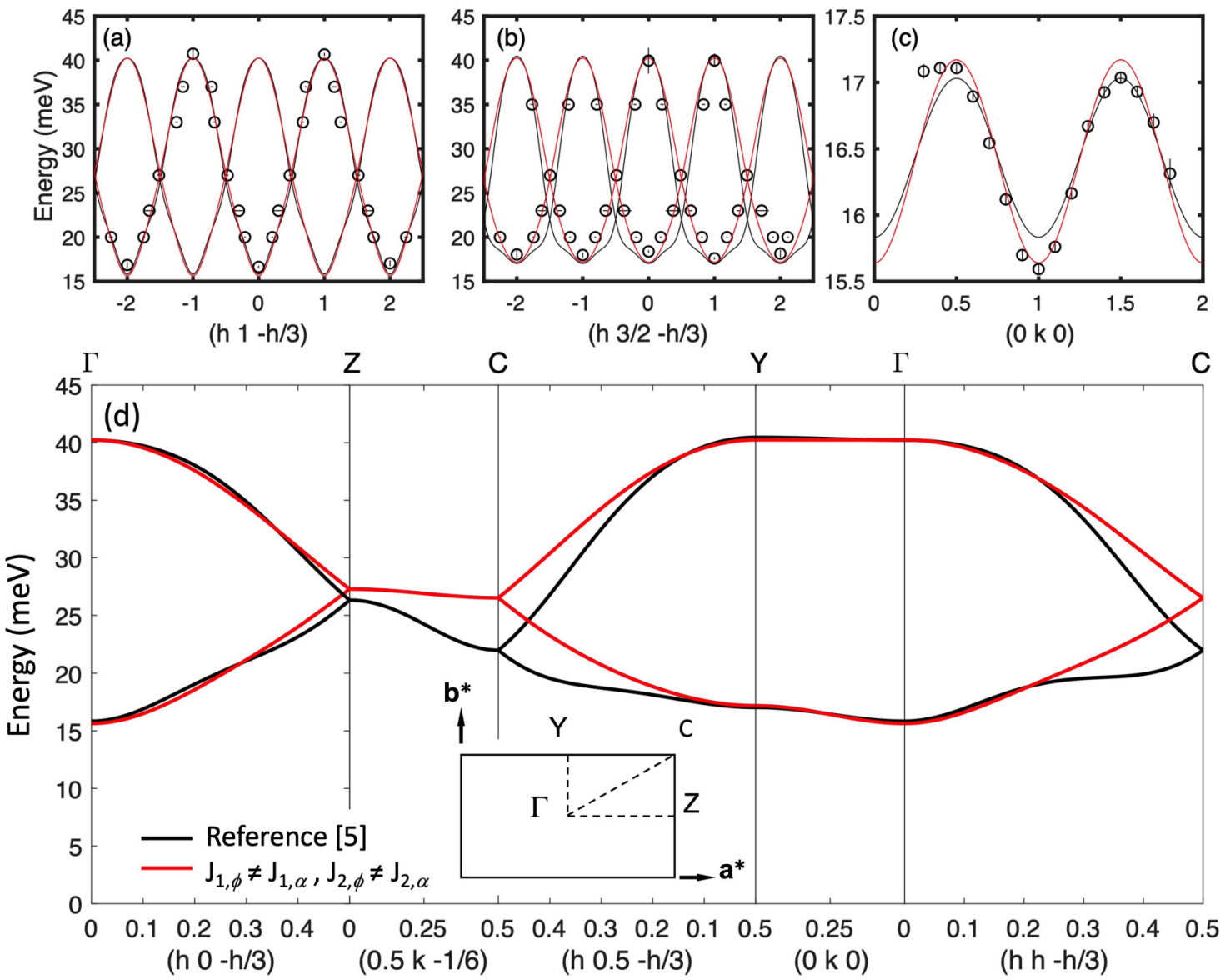

FIG. 4. The fitted data and dispersion curves for slices along different reciprocal space directions for FePS 3 . The black lines show the dispersions following the analysis from Lançon et al. ${ }^{5}$ The red lines show the dispersions after allowing for a biquadratic interaction in the fitting, i.e., $J_{1, \phi} \neq J_{1, \alpha}$ and $J_{2, \phi} \neq J_{2, \alpha}$. The data points in (a)-(c) were determined by taking linear cuts through Figs. 2(b), 2(c), and 3(a), respectively. (d) The calculated dispersion over a selection of reciprocal space directions, with the inset showing the Brillouin zone in the $\left(a^{*} b^{*}\right)$ plane. 
TABLE I. Exchange parameters and anisotropy determined from fitting Eq. (5) with various constraints on the matrix elements in Eq. (4). All the fit parameters are expressed in meV. The fit quality, given by $\chi^{2}$, is also tabulated for the respective fits,.

\begin{tabular}{|c|c|c|c|c|c|c|c|}
\hline & $\Delta$ & $J_{1}$ & $2 \tilde{K}_{1} S^{2}$ & $J_{2}$ & $2 \tilde{K}_{2} S^{2}$ & $J_{3}$ & $\chi^{2}$ \\
\hline$J_{1, \phi}=J_{1, \alpha}, J_{2, \phi}=J_{2, \alpha}\left(\right.$ cf. Lançon et al. $\left.{ }^{5}\right)$ & 2.66 & 1.46 & & -0.04 & & -0.96 & 147 \\
\hline$J_{1, \phi} \neq J_{1, \alpha}, J_{2, \phi}=J_{2, \alpha}$ & 2.53 & 0.9 & 0.55 & -0.047 & & -0.64 & 57.7 \\
\hline$J_{1, \phi}=J_{1, \alpha}, J_{2, \phi} \neq J_{2, \alpha}$ & 2.46 & 1.46 & & -0.22 & 0.2 & -0.55 & 57.4 \\
\hline$J_{1, \phi} \neq J_{1, \alpha}, J_{2, \phi} \neq J_{2, \alpha}$ & 2.52 & 0.98 & 0.46 & -0.155 & 0.085 & -0.45 & 49 \\
\hline
\end{tabular}

are shown in Table I. The nearest-neighbor exchange, $J_{1}$, is ferromagnetic, the second nearest-neighbor exchange, $J_{2}$, is very close to zero, and the zig-zag magnetic structure for $\mathrm{FePS}_{3}$ is stabilized by a relatively strong antiferromagnetic $J_{3} .{ }^{16,18}$ The interlayer exchange, determined from separate data measured using a three-axis spectrometer, ${ }^{5}$ was found to be very small, $J^{\prime}=-0.0073(3) \mathrm{meV}$. Its inclusion has only a very minor effect on modeling the spin waves and it will be ignored in subsequent discussion.

The dynamic structure factors were calculated with these parameters, and they are shown in Figs. 2(d)-2(f), corresponding to the data in Figs. 2(a)-2(c), respectively, and Figs. 3(c) and 3(d), corresponding to the data in Figs. 3(a) and 3(b), respectively. The expected spin wave contributions from the twinned domains are also plotted in these figures as white dashed lines. Inspection of the figures shows reasonable agreement between the neutron data and the calculated $S_{\text {mag }}(\mathbf{Q}, \omega)$; however; there are numerous discrepancies. The data and the model agree particularly well between Figs. 2(b) and 2(e), showing slices through Brillouin zone centers. The same is true when comparing the data and models in Figs. 3(a) and 3(c) and Figs. 3(b) and 3(d). However, comparison between the data and the model in Figs. 2(a) and 2(d) and Figs. 2(c) and 2(f) are not as good. The data are far more symmetric about the mid-point of the dynamic range for the spin waves at $\sim 27 \mathrm{meV}$ than the model calculations. The lowest energy spin waves, at $\sim 15 \mathrm{meV}$ at the Brillouin zone centers, are more rounded in the modeling than are apparent in the data. Furthermore, while both the data and the model in Figs. 2(c) and 2(f) show two intersecting spin-wave branches, the point of intersection in the data is at $\sim 27 \mathrm{meV}$ while it is at a lower energy of $\sim 20 \mathrm{meV}$ in the model.

Discrepancies are also apparent on comparing the expected contributions from the twinned domains. These contributions are most clearly seen in Fig. 2(b) as dispersive modes that cross at $h=0$ and $\omega=27 \mathrm{meV}$, in Fig. 3(a) as a steeply rising dispersive mode originating at $k=0.5$, and a mode in Fig. 3(b) descending from $\sim 30 \mathrm{meV}$ at $k \sim 1.7$ to $17 \mathrm{meV}$ at $k=2.5$. These contributions are not reproduced well by the modeling, which tend to shift them to lower energies.

The fits were substantially improved by allowing for $J_{1, \phi} \neq$ $J_{1, \alpha}$ and/or $J_{2, \phi} \neq J_{2, \alpha}$. Table I shows the fit parameters, presented in a form consistent with Eq. (7). The table includes a column showing a goodness-of-fit expression, $\chi^{2}$, for the different fits to the spin waves. Three fits with different non-equal exchanges were performed in total. The value for $\chi^{2}$ decreased substantially by a factor $\sim 3$ on allowing either $J_{1, \phi} \neq J_{1, \alpha}$ or $J_{2, \phi} \neq J_{2, \alpha}$ while constraining the other exchanges to be equal. Allowing both to be unequal further decreased $\chi^{2}$ by $\sim 15 \%$. The last fits are shown as red lines in Figs. 4(a)-4(c) and the calculated $S(\mathbf{Q}, \omega)$ for the corresponding parameters are shown for the respective slices through reciprocal space in Figs. 2(g)-2(i), 3(e), and 3(f), although it was barely possible to differentiate between similar plots using the parameters when either the first or second nearest-neighbor exchanges were constrained to be equal. Inspection of the figures shows a much better agreement between the data and the calculations with the new parameters. The same is true when comparing the contributions from the twinned domains in Figs. 2 and 3.

Figure $4(\mathrm{~d})$ shows the calculated spin-wave dispersions over the whole Brillouin zone in the $\left(\mathbf{a}^{*}, \mathbf{b}^{*}\right)$ planes for both the parameters determined by Lançon et al. and the parameters from the new fits. The biggest differences are seen along the $\mathrm{Z}-\mathrm{C}$ Brillouin zone boundary, where the spin waves become almost dispersionless when the exchange interactions for equivalent nearest-neighbor distances are not constrained to be equal.

Comparison of the values in Table I shows that the magnitude of the single-ion anisotropy does not vary greatly between the fits. This is expected, as the anisotropy is the dominant factor behind the observed spin-wave gap of $\sim 15 \mathrm{meV}$ and is not multiplied by a q-dependent term in Eq. (4). Allowing for non-equal $J_{1}$ resulted in substantially different values with one of the two exchange parameters being close to that determined by Lançon et al. and the other being significantly smaller by $\approx 1 \mathrm{meV}$. The same is true when fitting with non-equal $J_{2}$ with a smaller absolute difference between the two parameters of $0.2-0.4 \mathrm{meV}$ but a much larger relative difference. The value for $J_{3}$ decreased by $\approx 30 \%$ for non-equal $J_{1}$ and/or $J_{2}$.

\section{DISCUSSION}

The constraints implicit in the assumptions adopted by Lançon et al. in their analysis ${ }^{5}$ were that the lattice parameters were related through the expressions $b=\sqrt{3} a$ and $a=-3 c \cos \beta$ and that the fractional coordinates for the $\mathrm{Fe}^{2+}$ ions were $\left(0, \frac{1}{3}, 0\right)$, meaning that the magnetic lattice formed a perfect honeycomb and that the exchange pathways were identical for all corresponding nearest-neighbors. Inspection of the crystal structure for $\mathrm{FePS}_{3}$ shows this to be a very good assumption, ${ }^{1}$ with the constraints on the lattice parameters being correct to better than $0.05 \%$ and the constraint on the fractional coordinates being correct to better that $3 \%$. The assumptions also require that the exchange pathways, mediated by the sulfur atoms and with a possible contribution by the phosphorus, between nominally equivalent nearest-neighbors are also equal. Again, inspection of the crystal structure shows this 
to be a good assumption. $\mathrm{FePS}_{3}$ is only very slightly distorted from being an antiferromagnet with a perfect honeycomb lattice.

Equation (4) requires that the $\mathrm{Fe}^{2+}$ ions are on a perfect honeycomb in that the vectors connecting the interlocking Bravais lattices are constructed from rational fractions of the magnetic basis vectors. Nonetheless, they will allow for deviations from a perfect structure in that the exchanges, shown in Fig. 1, connect Fe atoms with equivalent nearest-neighbor distances in the distorted honeycomb. A difference between, for example, $J_{1, \phi}$ and $J_{1, \alpha}$ in the fits could reflect differences in the nearest-neighbor distances and small distortions in the bond angles with the sulfur atoms that would mediate the exchange. However, the degree of the structural distortion would seem to be far too small to result in a difference of $\approx 1 \mathrm{meV}$ observed in Table I. The assumption is supported by $a b$ initio calculations ${ }^{22,23}$ which calculated magnetic exchange parameters for energetically relaxed structures. Consistent with observations, the calculated structures were only very slightly distorted and the calculations gave unique exchange parameters for nominally equivalent nearestneighbors which, depending on the magnitude of the on-site repulsion term, were comparable to those of Lançon et al.

It appears more likely that there is another important energy term in the magnetic Hamiltonian for $\mathrm{FePS}_{3}$. The biquadratic term is a suitable candidate for allowing differences between nominally equivalent nearest-neighbors through the introduction of the biquadratic exchange parameter, $\tilde{K}$. Biquadratic exchange interactions can occur in Mott insulators with spins $S \geq 1$ from fourth-order perturbation processes in electron hopping. ${ }^{24,25}$ The biquadratic coupling may be further enhanced by metallicity ${ }^{19,20}$ or by substantial magnetoelastic interactions. ${ }^{26}$ The latter effect is particularly applicable for $\mathrm{FePS}_{3}$. The onset of magnetic order at the Néel temperature is accompanied by a discontinuous change in the temperaturedependence of the lattice parameters ${ }^{2,21}$ leading to the conclusion that the transition is of first order. Furthermore, $\mathrm{FePS}_{3}$ shows strong magnetoelastic effects at high magnetic fields to the point where a sample will self-delaminate when a sufficiently high field is applied along the $\mathbf{b}$ direction. ${ }^{18}$ Consequently, the magnetoelastic effect may be responsible for the large values of the biquadratic constant $\tilde{K}$ found from fitting the experimental magnon dispersion.

The inclusion of the biquadratic term does not lead to a complete understanding of the magnetic dynamics of $\mathrm{FePS}_{3}$. The highfield magnetization measurements showed a high degree of anisotropy when the field was applied in the $a b$ planes, being normal to the ordered moment direction, ${ }^{18}$ which would not be observed for dynamics governed by Eq. (6). The observed anisotropy is presumed to be due to the specifics of the coupling between the spin wave and phonon dynamics which are not captured with the simple biquadratic term.

Furthermore, the application of a sufficiently high magnetic field along the ordered moment direction led to an intermediate partially magnetized ordered state before being driven to a fully magnetized state. Inspection of a calculated phase diagram for an Ising two-dimensional honeycomb system with exchange interactions up to the third nearest-neighbor showed that the exchange parameters from Lançon et al. would not give rise to such a partially magnetized state, ${ }^{18}$ which requires that $J_{2} \leq-J_{1} / 2$. The inclusion of the biquadratic term will not solve this discrepancy. Not only do the parameters in Table I show that $J_{2,\{\alpha, \phi\}}>$
$-J_{1,\{\alpha, \phi\}} / 2$ for all combinations of $\{\alpha, \phi\}$, the effective exchange given in Eq. (7) is only applicable for linear spin waves and must not be used to calculate the energies of the magnetic structures. The proposed solution to the discrepancy was to adapt Eq. (1) to become an XXZ Hamiltonian. ${ }^{18}$ The exchange parameters found from neutron spectroscopy would be consistent with the exchange parameters acting on the $S_{i}^{x} S_{j}^{x}$ product, while the magnetic structure would be determined with the exchange parameters acting on $S_{i}^{z} S_{j}^{z}$. The solution is coherent with the introduction of a biquadratic term considered here as only the spin-wave dynamics are considered, although future efforts will be made to investigate the need for an anisotropic $\tilde{K}$ in the Hamiltonian.

There is some ambiguity in the fits concerning whether unequal exchanges are required for $J_{1}$ only, for $J_{2}$ only, or for both. The quality of the fits is essentially the same if either $J_{1}$ or $J_{2}$ are allowed to be unequal, and it is this allowance that gives the greatest improvement over the findings from Lançon et al. Allowing both to be unequal did improve the quality of the fits, but not so much as to be conclusive. It is likely that $J_{1}$ is the most important exchange to be unequal. The exchange paths between the $\mathrm{Fe}^{2+}$ ions will be mostly mediated by the sulfur atoms. Inspection of the crystal structure shows that the exchange paths between nearest-neighbors are straight forward, but that exchange paths to the second nearest-neighbors are convoluted. $^{27,28}$ It seems probable that $J_{2}$ remains close to zero and that $J_{1}$, which is strong, is the exchange most affected.

A final observation is of interest in the spin dynamics for $\mathrm{FePS}_{3}$. The data, and now the modeling, establish that the spin waves along the $\mathrm{Z}-\mathrm{C}$ boundary consist of two approximately linear branches that cross. As shown in Fig. 4(d), the crossing point is almost dispersionless, varying by $\sim 1 \mathrm{meV}$ from the $\mathrm{Z}$ to the $\mathrm{C}$ points. The spin-wave dispersion is thus consistent with the presence of two-dimensional Dirac magnon nodal lines, as described by Owerre. ${ }^{29}$ Dirac points, defined as a point at which valence and conduction bands for a two-dimensional conductor form cones that touch at their apexes, feature in the description of the electron transport in graphene. The points have an associated chirality to their Berry curvature that leads to magnetoelectric effects that can be exploited in technology. Recent studies have searched for magnetic analogs in the spin dynamics of layered honeycomb ferromagnets such as $\mathrm{CrX}_{3}(\mathrm{X}=\mathrm{Br}, \mathrm{Cl}, \mathrm{I}),{ }^{30}$ which may lead to applications in spintronics. The Dirac magnon points in these compounds are found in the Brillouin zone corners, where twodimensional magnon branches with linear dispersions cross. Owerre has proposed that these Dirac points can be generalized to nodal lines and have associated topological characteristics. The lines give rise to a peak in the magnon density of states. The density of states has been measured for FePS $3 .{ }^{14}$ Close inspection of the data shows a small feature at slightly less than $30 \mathrm{meV}$, corresponding to the energies of the magnon modes intersecting along the $\mathrm{Z}-\mathrm{C}$ boundary, consistent with the prediction of Owerre. More detailed studies of the magnetic dynamics in $\mathrm{FePS}_{3}$ are thus of interest for possible topological effects.

\section{CONCLUSIONS}

Neutron spectroscopy data showing the spin waves in $\mathrm{FePS}_{3}$ have been reanalyzed, allowing for unequal exchange parameters 
between nominally equivalent nearest-neighbors. The results show that substantial differences arise between ferromagnetically and antiferromagnetically coupled neighbors. The differences appear to be too large to be due to structural distortions, as $\mathrm{FePS}_{3}$ is very close to having a perfect honeycomb lattice, and instead can be explained with the introduction of a biquadratic exchange term into the Hamiltonian. The biquadratic term causes nominally equivalent sites to become inequivalent. It can be attributed to a strong magnetoelastic coupling, which causes the magnetic ordering transition to be of first order and is in evidence in magnetometry experiments, where the magnetostriction is so strong that the sample will delaminate when a sufficiently high field is applied along the $\mathbf{b}$ axis.

\section{ACKNOWLEDGMENTS}

The neutron experiment at the ISIS Neutron and Muon Source was supported by a beam time allocation from the Science and Technology Facilities Council (UK). D.L. acknowledges funding by the Swiss National Science Foundation, Grant Nos. 146870 and 166298, and the Sinergia Network MPBH, Grant No. 160765. D.L. and A.R.W. thank Professor H. Rønnow for stimulating discussions.

\section{DATA AVAILABILITY}

Raw data were generated by the MERLIN instrument at the ISIS Neutron and Muon Source, UK (experiment No. RB1410242). Derived data supporting the findings of this study are available from the corresponding author upon reasonable request.

\section{REFERENCES}

${ }^{1}$ G. Ouvrard, R. Brec, and J. Rouxel, Mater. Res. Bull. 20, 1181 (1985).

${ }^{2}$ P. Jernberg, S. Bjarman, and R. Wäppling, J. Magn. Magn. Mater. 46, 178-190 (1984).

${ }^{3}$ P. A. Joy and S. Vasudevan, Phys. Rev. B 46, 5425 (1992).

${ }^{4}$ K. Kurosawa, S. Saito, and Y. Yamaguchi, J. Phys. Soc. Jpn. 52, 3919-3926 (1983).

${ }^{5}$ D. Lançon, H. C. Walker, E. Ressouche, B. Ouladdiaf, K. C. Rule, G. J. McIntyre, T. J. Hicks, H. M. Rønnow, and A. R. Wildes, Phys. Rev. B 94, 214407 (2016).

${ }^{6}$ R. Brec, Solid State Ionics 22, 3 (1986).

${ }^{7}$ V. Grasso and L. Silipigni, Riv. Nuovo Cimento 25, 1 (2002).

${ }^{8}$ M. A. Susner, M. Chyasnavichyus, M. A. McGuire, P. Ganesh, and P. Maksymovych, Adv. Mater. 29, 1602852 (2017).

${ }^{9}$ F. Wang, T. A. Shifa, P. Yu, P. He, Y. Liu, F. Wang, Z. Wang, X. Zhan, X. Lou, F. Xia, and J. He, Adv. Funct. Mater. 28, 1802151 (2018).
${ }^{10}$ M. J. Coak, D. M. Jarvis, H. Hamidov, C. R. S. Haines, P. L. Alireza, C. Liu, S. Son, I. Hwang, G. I. Lampronti, D. Daisenberger, P. Nahai-Williamson, A. R. Wildes, S. S. Saxena, and J.-G. Park, "Tuning dimensionality in van-der-waals antiferromagnetic mott insulators TMPS3," J. Phys. Condens. Matter 32, 124003 (2019).

${ }^{11}$ Y. Wang, J. Ying, Z. Zhou, J. Sun, T. Wen, Y. Zhou, N. Li, Q. Zhang, F. Han, Y. Xiao, P. Chow, W. Yang, V. V. Struzhkin, Y. Zhao, and H.-K. Mao, Nat. Commun. 9, 1914 (2018).

${ }^{12}$ L. Onsager, Phys. Rev. 65, 117 (1944).

${ }^{13}$ J.-U. Lee, S. Lee, J. H. Ryoo, S. Kang, T. Y. Kim, P. Kim, C.-H. Park, J.-G. Park, and H. Cheong, Nano Lett. 16, 7433-7438 (2016).

${ }^{14}$ A. R. Wildes, K. C. Rule, R. I. Bewley, M. Enderle, and T. J. Hicks, J. Phys. Condens. Matter 24, 416004 (2012).

${ }^{15}$ E. M. Wheeler, R. Coldea, E. Wawrzyńska, T. Sörgel, M. Jansen, M. M. Koza, J. Taylor, P. Adroguer, and N. Shannon, Phys. Rev. B 79, 104421 (2009).

${ }^{16}$ E. Rastelli, A. Tassi, and L. Reatto, Physica B 97, 1-24 (1979).

${ }^{17}$ E. Rastelli, A. Tassi, and L. Reatto, J. Magn. Magn. Mater. 15-18, 357-358 (1980)

${ }^{18}$ A. R. Wildes, D. Lançon, M. K. Chan, F. Weickert, N. Harrison, V. Simonet, M. E. Zhitomirsky, M. V. Gvozdikova, T. Ziman, and H. M. Rønnow, "High field magnetization of FePS 3 ," Phys. Rev. B 101, 024415 (2020).

${ }^{19}$ A. L. Wysocki, K. D. Belashchenko, and V. P. Antropov, Nat. Phys. 7, 485-489 (2011).

${ }^{20}$ A. L. Wysocki, K. D. Belashchenko, L. Ke, M. van Schilfgaarde, and V. P. Antropov, J. Phys. Conf. Ser. 449, 012024 (2013).

${ }^{21}$ C. Murayama, M. Okabe, D. Urushihara, T. Asaka, K. Fukuda, M. Isobe, K. Yamamoto, and Y. Matsushita, J. Appl. Phys. 120, 142114 (2016).

${ }^{22}$ B. L. Chittari, Y. Park, D. Lee, M. Han, A. H. MacDonald, E. Hwang, and J. Jung, "Electronic and magnetic properties of single-layer $m \mathrm{PX}_{3}$ metal phosphorous trichalcogenides," Phys. Rev. B 94, 184428 (2016).

${ }^{23}$ Y. Gu, Q. Zhang, C. Le, Y. Li, T. Xiang, and J. Hu, "Ni-based transition metal trichalcogenide monolayer: A strongly correlated quadruple-layer graphene," Phys. Rev. B 100, 165405 (2019).

${ }^{24}$ F. Mila and F.-C. Zhang, Eur. Phys. J. B 16, 7-10 (2000).

${ }^{25} \mathrm{~K}$. Tanaka, Y. Yokoyama, and C. Hotta, "Origin of biquadratic exchange interactions in a mott insulator as a driving force of spin nematic order," J. Phys. Soc. Jpn. 87, 023702 (2018)

${ }^{26} \mathrm{~K}$. Penc, N. Shannon, and H. Shiba, "Half-magnetization plateau stabilized by structural distortion in the antiferromagnetic heisenberg model on a pyrochlore lattice," Phys. Rev. Lett. 93, 197203 (2004).

${ }^{27}$ G. L. Flem, R. Brec, G. Ouvard, A. Louisy, and P. Segransan, "Magnetic interactions in the layer compounds $\mathrm{MPX}_{3}(\mathrm{M}=\mathrm{Mn}, \mathrm{Fe}, \mathrm{Ni} ; \mathrm{X}=\mathrm{S}, \mathrm{Se})$," J. Phys. Chem. Solids 43, 455-461 (1982), ISSN 0022-3697.

${ }^{28}$ D. Lançon, R. A. Ewings, T. Guidi, F. Formisano, and A. R. Wildes, "Magnetic exchange parameters and anisotropy of the quasi-two-dimensional antiferromagnet NiPS 3 ," Phys. Rev. B 98, 134414 (2018).

${ }^{29}$ S. A. Owerre, Sci. Rep. 7, 6931 (2017).

${ }^{30}$ S. S. Pershoguba, S. Banerjee, J. C. Lashley, J. Park, H. Ågren, G. Aeppli, and A. V. Balatsky, "Dirac magnons in honeycomb ferromagnets," Phys. Rev. X 8, 011010 (2018). 